\title{
Stability of giant vortices in quantum liquids
}

\author{
Christophe Josserand \\ Laboratoire de Modélisation en Mécanique, \\ CNRS UMR 760\%, 8 rue du Capitaine Scott, \\ 75015 Paris-France
}

\begin{abstract}
We show how giant vortices can be stabilized in strong external potential Bose-Einstein condensates. We illustrate the formation of these vortices thanks to the relaxation Ginzburg-Landau dynamics for two typical potentials in two spatial dimensions. The giant vortex stability is studied for the particular case of the rotating cylindrical hard wall. The minimization of the perturbed energy is simplified into a one dimensional relaxation dynamics. The giant vortices can be stabilized only in a finite frequency range. Finally we obtain a curve for the minimum frequency needed to observe a giant vortex for a given nonlinearity.
\end{abstract}

Keywords: Bose-Einstein condensates, quantized vortex, multiple charge, energy minimization.

Quantized vortices are characteristics of quantum systems. They appear for instance when a rotation is imposed to a quantum liquid. We study here the conditions under which vortices with multiple charges can be observed. It is in general not possible since the energy of an array of single vortices is below the energy of a multiple vortex of same total charge. However an opposite trend appears for the energy if the system is confined. This in particular the case for the recent achieved Bose-Einstein condensates where an optical trap maintains the gas in a small volume. We show that if the trap potential is strong enough the multiple charge vortex becomes stable. The linear stability of these giant vortices is performed using one dimensional minimization techniques.

\section{INTRODUCTION}

The recent achievement of Bose-Einstein condensates (BEC) 1, 2, 3] has strongly renewed and restimulated the numerous works and theories on quantum gases/liquids. By their lower densities, their larger healing lengthes, the BEC present important advantages compared to the traditional Helium superfluids, regardless the fundamental interest of the Bose-Einstein transition itself. One of the striking properties of these quantum gases/liquids is the 
quantization of the vorticity 4]. It manifests through the formation of vortices whose circulation is quantified in units of $\hbar / m$, where $m$ is the mass of an atom of the gas and $\hbar$ is the Planck constant. Such vortices have been observed in BEC by two different procedures these last years [5, 6, 7]. Firstly [5] by rotating the BEC through laser techniques. This experiment is the analogy of the famous rotating bucket of superfluid helium where the first direct observation of quantized vortices was obtained [8]. The vortices appear suddenly as the rotation frequency increases, either one by one, or by group, depending on the experimental procedure. The other experiment 6 , 7$]$ exploits the dynamical transition to vortex shedding of an accelerated superflow $[9]$. Experimentally, to achieve the BE condensation the atoms of the condensate have to be placed in an optical trap which creates an effective (mostly harmonic) attractive potential. Thus, in the above experiments only single charged vortices were observed. No multicharged vortices (called giant vortex of charge $q$ or $q$-vortex later on) were detected. However, by imposing specific initial states, it has been possible to observe metastable long-lived giant vortices experimentally [10]. It is in fact well known that multicharged vortices are always dynamically and thermodynamically unstable in homogenous and infinitely large quantum liquids [1]]. When the system is submitted to a trap potential, the situation becomes more tricky since the potential encourages vortex merging while the vortices try to expand the condensate. For BEC in harmonic trap, variational stability analysis suggests indeed the existence of a critical frequency above which the giant vortex should be energetically favoured to the single vortices lattice 12]. But this frequency is found higher than the frequency of the quadratic trapping potential. At such speed, the condensate is unstable since the centrifugal force overcomes the trap confinement. This explains why no stable giant vortices have been observed until now in rotating BEC, where only harmonic trap were experimentally considered. This peculiar property of harmonic confinement does not actually prevent the stability of giant vortices for stronger trap potentials. In fact different groups have shown numerically and argued analytically that giant vortices are stable for high enough rotation if the trap potential is taken stronger than harmonic $13,|14,15|$. Numerical simulations have exhibited these giant vortices and some coexistence diagramms were even obtained [13, 15], although no clear stability arguments were presented. In the other work[14] the stability analysis was only performed in the framework of Wigner-Seitz approximation of a vortex lattice. Adequate strong trap potentials can be experimentally achieved thanks for example to Laguerre-Gaussian laser beams $[16\rfloor$ but the observation of stable giant vortices remains still to be done.

In this note, we address the stability analysis of the giant vortices for the rotating cylinder. We use numerical simulations for two-dimensional systems subject to rotation $\boldsymbol{\Omega}=\Omega \mathbf{z}$, assuming translational invariance in the $z$ - 
direction. The giant vortex formation is shown for illustration in a rotating BEC with quartic potential but we restrict thereafter our treatment to the case of an infinite cylindrical potential.

\section{THE MODEL}

The dynamics of a quantum Bose gas in the rotating frame in two dimensions is described through the dimensionless Gross-Pitaevskiǐ (G-P) equation [17, 18, 19]:

$$
i \partial_{t} \psi=\left(-\frac{1}{2} \nabla^{2}+V(r)+M|\psi|^{2}+i \Omega \partial_{\theta}\right) \psi
$$

where $\psi(\mathbf{r}, t)$ is the condensate wave function normalized to unity: $\int d \mathbf{r}|\psi|^{2}=1$. $\mathbf{r}$ is the position vector $(x, y), r=|\mathbf{r}|$ and $\theta$ the corresponding cylindrical coordinates. The nonlinear strength $M=4 \pi N a$ is related to the product of the particle number per unit length $N$ with the s-wave scattering length $a$. This dimensionless equation has been in fact deduced from the usual model using a typical length scale $d$ that is defined by the trap properties. Consequently the length, energy and time in equation (1) are given in units of $d, \hbar^{2} /\left(m d^{2}\right)$ and $m d^{2} / \hbar(\hbar$ the Planck constant and $m$ the particle mass). The usual harmonic potential $\frac{1}{2} m \omega_{t}^{2} r^{2}$ leads to $V(r)=\frac{1}{2} r^{2}$ with $d=\sqrt{\hbar /\left(m \omega_{t}\right)}$. Below we restrict

our work to only two traps geometry. Firstly for illustration a quartic potential $\frac{m^{2} \omega_{t}^{3} r^{4}}{\hbar}$, so that $V(r)=\frac{1}{4} r^{4}$ and $d=\sqrt{\hbar /\left(m \omega_{t}\right)}$ again. This potential has a smooth behavior and describes a stronger trap potential than the usual harmonic potential of the experiments. The other potential models a quantum liquid entrapped in a cylindrical bucket of radius $L$. With $d=L$ the potential is described by $V(r)=0$ if $r<1$ and $V(r)=\infty$ otherwise. It corresponds to the well-known experiment on superfluid Helium where quantized vortices have been first vizualized $[8]$. Note that equation (1) admits only two control parameters for a given potential: the interaction coefficient $M$ and the angular frequency $\Omega$. The dynamics derives from a free energy $F$ through: $\imath \partial_{t} \psi=\frac{\delta F}{\delta \psi^{*}}$ with:

$$
F=\int d \mathbf{r}\left(\frac{1}{2}|\nabla \psi|^{2}+i \Omega \psi^{*} \partial_{\theta} \psi+V(r)+\frac{M}{2}|\psi|^{4}\right)
$$

Stable equilibrium solutions of Eq. (1) correspond to local minima of $F$. The ground state is determined among the stable equilibrium solution as the one with the lowest energy. Stable equilibrium states of the condensate can be reached numerically through the dissipative dynamics of the Ginzburg-Landau equation. Formally it corresponds to 
an imaginary time evolution of the GP equation (1):

$$
\partial_{t} \psi=-\left(-\frac{1}{2} \nabla^{2}+4 \pi N a|\psi|^{2}-\mu(N, \Omega)+V(r)+i \Omega \partial_{\theta}\right) \psi
$$

We have introduced the chemical potential $\mu$ as the Lagrangian multiplier ensuring that the norm of $\psi$ is unity. Numerical simulations have been performed in two space dimensions up to $256 \times 256$ grid points. We have checked that numerical precision is not significantly affected by higher spatial discretizations for our range of parameters. Both Fast Fourier Transform (FFT) or finite difference methods have been used for spatial derivatives and again no dramatic changes were noticed between these schemes. The relative error in the normalization of the wave function was controlled to be below $10^{-3}$ and the precision for $\mu$ is better than $10^{-3}$.

\section{GIANT VORTEX FORMATION}

To illustrate the formation of giant vortices, we follow numerically an equilibrium solution as $\Omega$ evolves. We start at $\Omega=0$ with the ground state (vortex free solution) and we increase suddenly $\Omega$ of a small increment $\delta \Omega$. We retrieve an equilibrium state through the imaginary time dynamics (3) and the procedure is then successively reproduced. Both the quartic and the hard-wall potentials are considered.

We first present the results for the quartic potential with $M=12 \pi$ (figures 1). Figure (1]a) shows the ground state solution, vortex-free at $\Omega=0$. This solution survives without any change until $\Omega=5.4$ where suddenly four

vortices enter into the cloud (figure1,b). They are well separated and form a square lattice. We observe also a spatial expansion of the cloud due to centrifugal effects. As $\Omega$ increases further on(figure 1 c)), the four vortices approach slowly the center of the trap. In this case the full merging of the four vortices is attained at $\Omega=7.2($ figure $1 \mathrm{~d})$ ), just before new vortices enter the cloud (at $\Omega=7.35$ ).

A similar situation is observed for a superfluid in a cylindrical trap although in that case the condensate cannot expand when vortices are nucleated. Indeed we account for the infinite hard wall potential by imposing as boundary conditions $\psi=0$ for $r \geq 1$. Figure (2) shows the density $|\psi|^{2}$ of the solution of equation (3) as $\Omega$ increases for $M=300$. The nucleation of four vortices occurs at $\Omega=14.8$ (figure (2) a)) from the vortex free solution. Further increments of $\Omega$ lead to the convergence of the four vortices towards the center of the trap(figure (2 b)). But a different scenario arises here compared to the dynamics observed for the quartic potential. Indeed, before the four 
a)

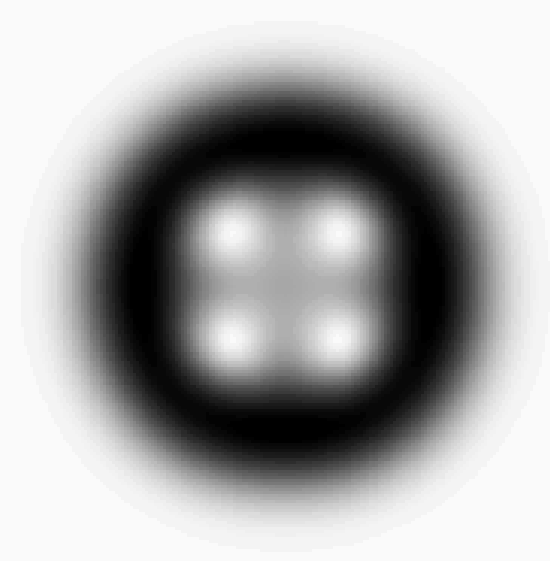

c)

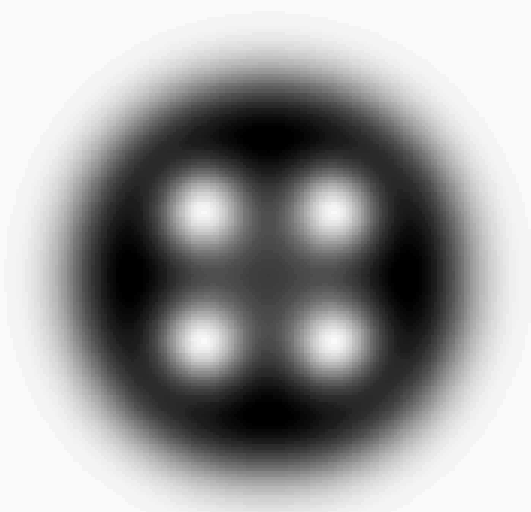

b)

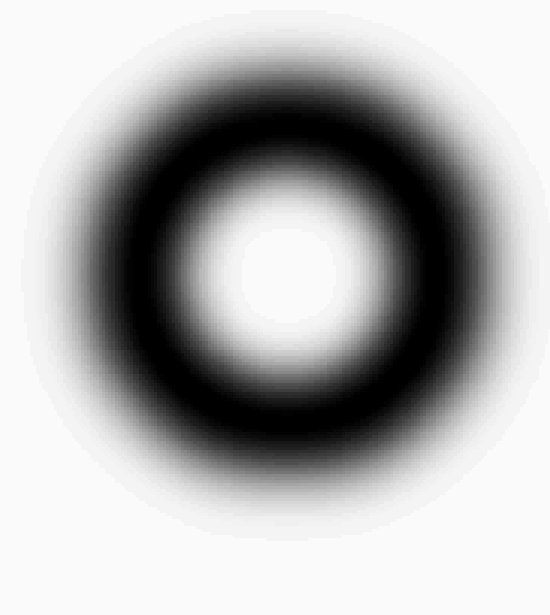

Figure 1: Quartic trap potential: density plots $|\psi|^{2}$ for $N \cdot a=3$ at $\Omega=$ a) 0 , b) 5.4, c) 6.6, and d) 7.2. The grid size is $128 \times 128$ and a pseudo-spectral method is used. The darker the color, the higher the density so that the vortices appear as white hole in the condensate.

vortices collapse in a single 4 -vortex, four new vortices are nucleated in the cloud at $\Omega=20.8($ figure $(2 \Omega$ c) $)$. The condensate is now composed of a lattice of eight well separated vortices. Then new increases of $\Omega$ lead eventually to the merging into a giant 8 -vortex at $\Omega=26.2$ (figure $(2 \mathrm{~d})$ ). Above this rotation, when new vortices are nucleated, they are immediatly absorbed by the giant vortex. This persistence of giant vortices for increasing frequencies has been in fact observed for all the numerical simulations that we have performed. 

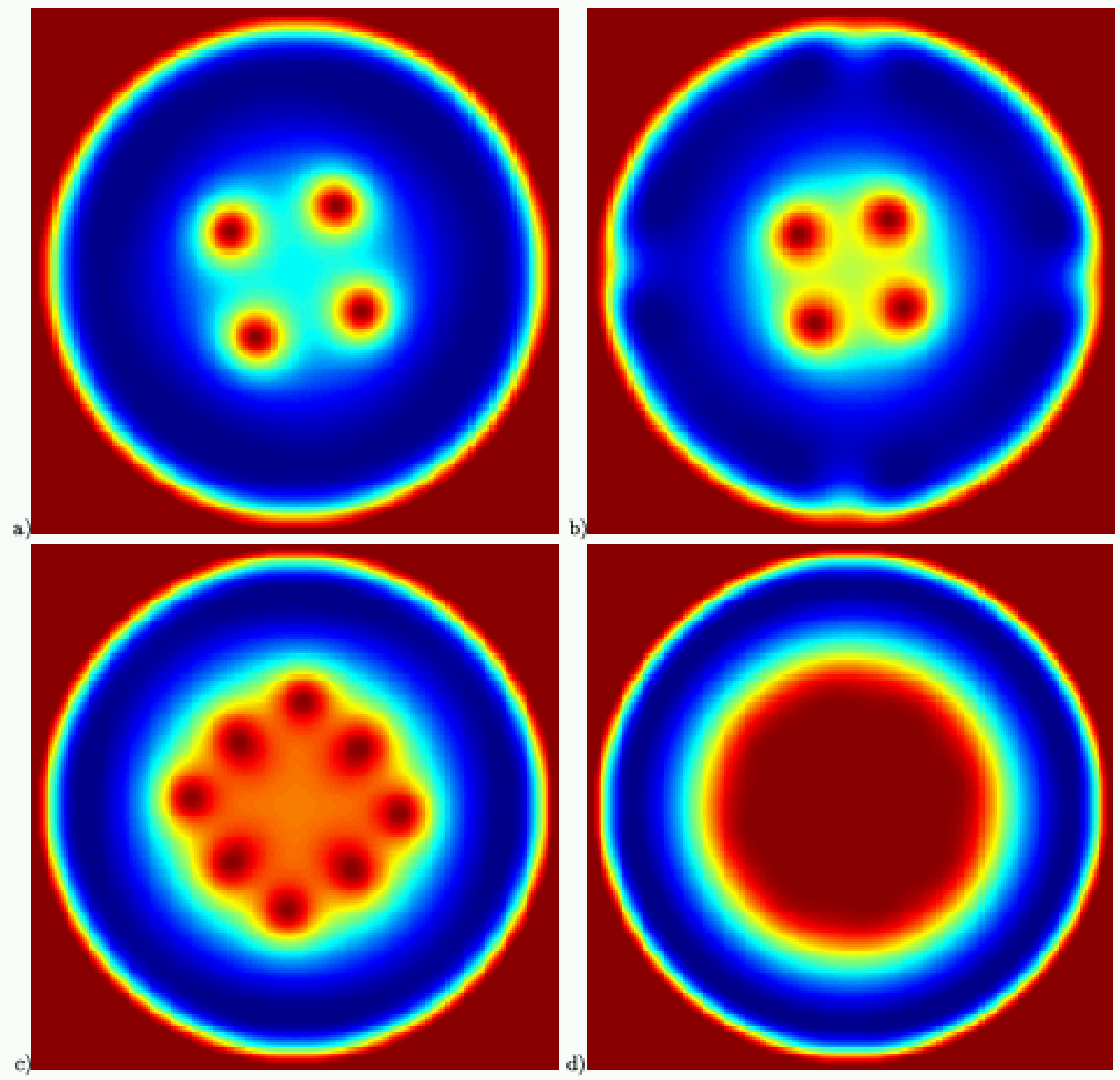

Figure 2: Density plots for $M=300$, for $\Omega=$ a) 14.8 , b) 18 ., c) 20.8 , and d) 26.2 . The grid size is $250 \times 250$ and the increment of the angular frequency is 0.2 . The color scale goes from blue for zero density to red for the maximum density. The vortices appear as blue holes on the density field.

\section{STABILITY ANALYSIS}

By sake of simplicity, the stability analysis is performed for the cylindrical wall trap potential. We want to address the conditions under which conditions a $q$-vortex is a local minimum of the free energy (2). We determine firstly the giant $q$-vortex state by seeking solutions of the form: 


$$
\psi_{q}^{0}(\mathbf{r})=f_{q}(r) e^{i q \theta}
$$

The trivial symetry of the problem $q \rightarrow-q$ with $\Omega \rightarrow-\Omega$ makes us only consider later on the case $q>0$. From the free-energy principle, to be an equilibrium solution $f_{q}$ has to satisfy:

$$
\frac{1}{2}\left(f_{q}^{\prime \prime}+\frac{f_{q}^{\prime}}{r}-\frac{q^{2}}{r^{2}} f_{q}\right)-M f_{q}^{3}+\left(\mu_{q}(M, \Omega)+q \cdot \Omega\right) f_{q}=0
$$

$\mu_{q}(M, \Omega)$ is introduced again as the Lagrangian multiplier for the norm constraint $<\psi_{q}^{0}\left|\psi_{q}^{0}>=<f_{q}\right| f_{q}>=$ $2 \pi \int r d r f_{q}^{2}=1$, where $<\cdot \mid \cdot>$ stands for the usual scalar product. The external potential term is replaced by the boundary condition $\psi=0$ for $r \geq 1$ so that this potential term is everywhere zero in the equation. We observe from the preceeding equation (4) that the giant vortex solution is independant of $\Omega$ which only intervenes in the coefficient $\mu_{q}(M, \Omega)=\mu_{q}^{0}(M)-q \cdot \Omega$. These solutions are thus equilibrium states for all frequencies $\Omega$ regardless their stability. The free energy of the vortex writes:

$$
F_{q}=2 \pi \int r \cdot d r\left(\frac{1}{2}\left(\left(f_{q}^{\prime}\right)^{2}+\frac{q^{2}}{r^{2}} f_{q}^{2}\right)-q \Omega f_{q}^{2}+\frac{M}{2} f_{q}^{4}\right)=T_{q}(M)+U_{q}(M)-q \cdot \Omega
$$

Where $T_{q}(M)=\pi \int r \cdot d r\left(\left(f_{q}^{\prime}\right)^{2}+\frac{q^{2}}{r^{2}} f_{q}^{2}\right)$ is the kinetic energy and $U_{q}(M)=M \pi \int r \cdot d r f_{q}^{4}$ the nonlinear energy term. $E_{q}(M)=T_{q}(M)+U_{q}(M)$ is the total energy of the vortex solution. The $q$-vortex function $f_{q}$ is found as before through the dissipative dynamics of the axisymetric Ginzburg-Landau equation:

$$
\partial_{t} f_{q}=\frac{1}{2}\left(f_{q}^{\prime \prime}+\frac{f_{q}^{\prime}}{r}-\frac{q^{2}}{r^{2}} f_{q}\right)-M f_{q}^{3}+\mu_{q}^{0} f_{q}
$$

so that the equilibrium solutions $f_{q}$ are local mimina of the free energy $F_{q}$. Figure (3a)) shows the density $f_{q}^{2}$ of the solution for $M=40 \pi$ and $q=0,1, \ldots, 10$ as a function of the position $r$. As it can be emphasized from equation (4), the bigger $q$, the larger is the core of the vortex, and the higher is the density level outside the vortex core. Indeed, equation (44) for $r \rightarrow 0$ implies that the solution $f_{q}$ behaves like $r^{q}$ near the center. Figure (3 b)) represents also the $q=5$ solution for $M$ varying between $4 \pi$ and $800 \pi$. The vortex core is shrinking as $M$ increases in agreement with the evolution of the condensate healing length $(\propto 1 / \sqrt{M})$, so does the wall boundary layer. 
a)
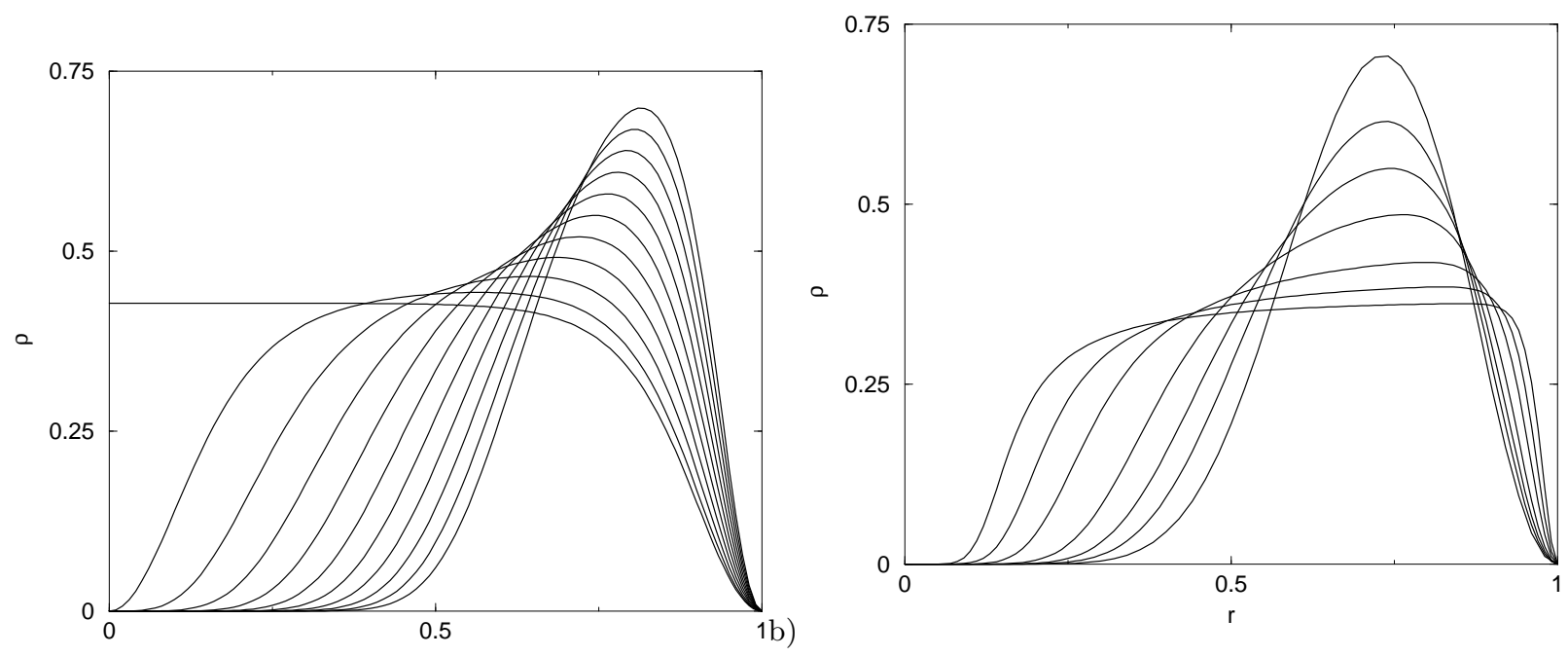

Figure 3: Density of the condensate $f_{q}^{2}$ for a) $M=40 \pi$ and $q=0,1 \ldots 10$ b) $q=5$ and $M / p i=4,20,40,80,200400$ and 800 ; the higher the maximum of the curve, the smaller $M$ and the bigger $q$. The profiles have been obtained as the stationnary solution of equation (6) and a regular grid of only 100 mesh points is needed for convergence.

Figure (4) shows the free energy of the giant vortices at $\Omega=0$ as a function of $q$ for different values of $M$. The energy is found convex with $q$ for each curve. From this curve we can determine for each $\Omega$ which $q$-vortex minimizes the free energy. It defines an increasing set of critical frequencies $\Omega_{q}^{m}(M)$ such that the $q$-vortex is the one with the lowest energy for $\Omega_{q-1}^{m}(M)<\Omega<\Omega_{q}^{m}(M)$ with

$$
\Omega_{q}^{m}(M)=E_{q+1}-E_{q}
$$

A $q$-vortex is stable when it is a local minimum of the free energy (21). We expect from the numerics that such a vortex is unstable at low enough frequency $\Omega$ : it decomposes itself into $q$ single vortices (see figures (11) and (2)). On the other hand, high frequencies destabilize the $q$-vortex by nucleating new vortices which gather into a new giant vortex as observed for $M=100$. Thus, the $q$-vortex can only be stable in a finite range of frequency and depending on the vaues of $q$ and $M$, we may observe that the $q$-vortex can never be stabilized (it is the case for $q=4, M=100$ ). The stability analysis is performed by an expansion of the free energy around the $q$-vortex solution. We write the perturbed wave-function of the giant vortex for $q>1$ in the form:

$$
\psi=\frac{\psi_{q}^{0}+\delta \psi}{\left\|\psi_{q}^{0}+\delta \psi^{q}\right\|}
$$

Following the periodic dependance in $\theta$, we decompose the perturbation $\delta \psi^{q}$ among azimuthal modes as a Fourier 


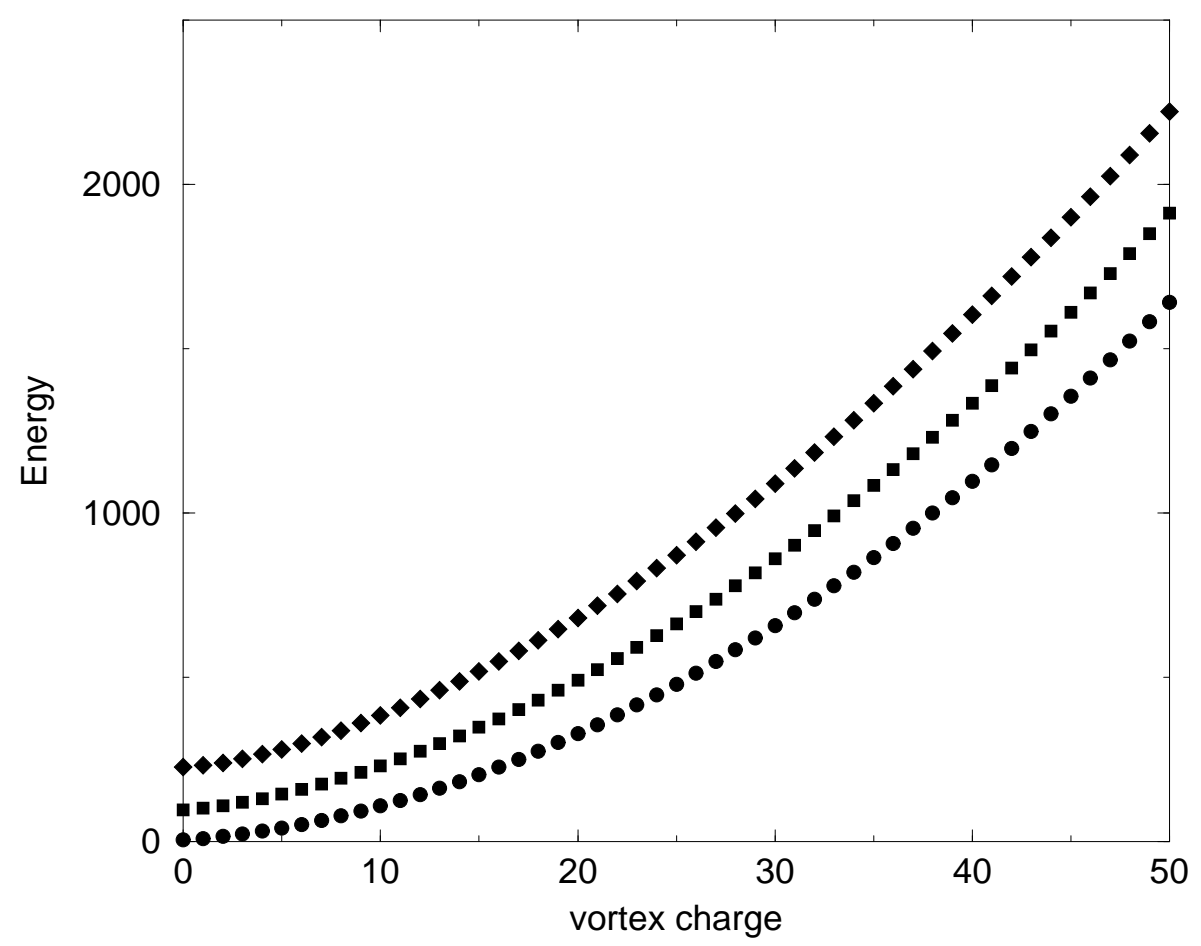

Figure 4: Energy of the vortex solutions as a function of the charge $q$ for three different values of $M$. From bottom to top,

$$
M=1 \text { (circles), } M=40 \text { (squares) and } M=100 \text { (diamonds). }
$$

set

$$
\delta \psi^{q}(r, \theta)=\sum_{q^{\prime}} \epsilon_{q^{\prime}} g_{q^{\prime}}^{q}(r) e^{i q^{\prime} \theta}
$$

The complex functions $g_{q^{\prime}}^{q}(r)$ describe the $q^{\prime}$ pertubation mode of the $q$ vortex and are normalized to unity. The amplitudes $\epsilon_{q^{\prime}}$ of the $q^{\prime}$ perturbation modes are complex numbers. We can omitt the $q$ perturbation mode which will bring nothing to the stability analysis since $\psi_{q}^{0}$ is already the minimizer of the axisymmetrical free energy with charge $q$. By analysing the perturbed wavefunction near $r=0$, we observe that for $q^{\prime}<q$ the perturbation mode describes the destabilization of the $q$ vortex into a $q^{\prime}$-vortex in the center surrounded by $q-q^{\prime}$ single vortices. On the other hand, for $q^{\prime}>q$ the perturbation describes the nucleation from the wall of $q^{\prime}-q$ isolated vortices. We obtain for the perturbed wavefunction:

$$
\psi=\frac{\psi_{q}^{0}+\sum_{q^{\prime} \neq q} \epsilon_{q^{\prime}} g_{q^{\prime}}^{q}(r) e^{i q^{\prime} \theta}}{\sqrt{1+\sum_{q^{\prime} \neq q}\left|\epsilon_{q^{\prime}}\right|^{2}}}
$$

The free energy correction $\delta F$ writes at the first non-zero order in the $\epsilon$ 's: 


$$
\delta F=\sum_{q^{\prime}<q^{\prime \prime}}\left(\epsilon_{q^{\prime}}, \epsilon_{q^{\prime \prime}}^{*}\right) \mathcal{L}_{q^{\prime}, q^{\prime \prime}}\left(\begin{array}{c}
\epsilon_{q^{\prime}}^{*} \\
\epsilon_{q^{\prime \prime}}
\end{array}\right)
$$

omitting the $q$ superscript from now on. The set of operators $\mathcal{L}_{q^{\prime}, q^{\prime \prime}}$ follows:

$$
\mathcal{L}_{q^{\prime}, q^{\prime \prime}}=\delta_{q^{\prime}+q^{\prime \prime}, 2 q}\left(\begin{array}{cc}
\delta F_{q^{\prime}} & 2 \pi M \int r f_{q}(r)^{2} g_{q^{\prime}}(r) g_{q^{\prime \prime}}(r) d r \\
2 \pi M \int r f_{q}(r)^{2} g_{q^{\prime}}^{*}(r) g_{q^{\prime \prime}}^{*}(r) d r & \delta F_{q^{\prime \prime}}
\end{array}\right)
$$

with the diagonal terms:

$$
\delta F_{q^{\prime}}=-T_{q}-2 U_{q}+\left(q-q^{\prime}\right) \Omega+2 \pi \int r d r\left(\frac{1}{2}\left(\left(g_{q^{\prime}}^{\prime}\right)^{2}+\frac{\left(q^{\prime}\right)^{2}}{r^{2}} g_{q^{\prime}}^{2}\right)+2 M \cdot g_{q^{\prime}}^{2} f_{q}^{2}\right)
$$

We notice here that the $\theta$ dependance of the free energy has been integrated so that further calculations will only concern the $r$ spatial variations. This axisymetrical simplification leads eventually to a one dimensional stability problem. The stability of the $q$-giant follows from the perturbated energy structure: if the operators $\mathcal{L}_{q^{\prime}, q^{\prime \prime}}$ are all found positive then the vortex is stable. On the other hand, if at least one of these operators is shown negative the giant vortex is unstable towards either decomposition into simpler vortices or nucleation/annihilation of vortices. The operators $\mathcal{L}_{q^{\prime}, q^{\prime \prime}}$ couple in fact the two modes $g_{q^{\prime}}$ and $g_{2 q-q^{\prime}}$ so that the determination of their full spectrum remains a complicate task.

However, if we could neglect the non-diagonal terms, the determination of the giant vortex stability would be straightforward. Indeed, the stability would be only determined by the minimization of the effective free energies $\delta F_{q^{\prime}}$ associated to the functions $g_{q^{\prime}}$ for each $q^{\prime} \neq q$. Following this assumption discussed in more details below, we only have to determine the eigenfunction $\hat{g}_{q^{\prime}}$ which minimizes the corresponding energy:

$$
\delta E_{q, q^{\prime}}=2 \pi \int r d r\left(\frac{1}{2}\left(\left(g_{q^{\prime}}^{\prime}\right)^{2}+\frac{\left(q^{\prime}\right)^{2}}{r^{2}} g_{q^{\prime}}^{2}\right)+2 M \cdot g_{q^{\prime}}^{2} f_{q}^{2}\right)
$$

with the following conditions (omitting the hat of the functions $\hat{g}_{q^{\prime}}$ later on):

$$
<g_{q^{\prime}} \mid g_{q^{\prime}}>=1 . \quad g_{0}^{\prime}(0)=0 \quad g_{q^{\prime} \neq 0}(0)=0 \quad g_{q^{\prime}}(1)=0
$$


We observe that energy (8) is unchanged through the symetry $q^{\prime} \rightarrow-q^{\prime}$ so that we just need to calculate the minimizer for $q^{\prime} \geq 0 . g_{q^{\prime}}$ corresponds in fact to the calculation of a $q^{\prime}$ giant vortex placed into the effective potential:

$$
U_{\text {eff }}(r)=2 M f_{q}(r)^{2}+V(r)
$$

The solution can be reached again through a dissipative dynamics:

$$
\partial_{t} g_{q^{\prime}}=\frac{1}{2}\left(g_{q^{\prime}}^{\prime \prime}+\frac{g_{q^{\prime}}^{\prime}}{r}-\frac{q^{2}}{r^{2}} g_{q^{\prime}}\right)-2 M f_{q}^{2} g_{q^{\prime}}+\mu^{\prime} g_{q^{\prime}}
$$

where $\mu^{\prime}$ is introduced here as the Lagrangian multiplier for the unity norm constraint. Figure (5) shows precisely different $g_{q^{\prime}}$ for $q=4$ and $M=300$. The $q$ giant vortex profile is also indicated. As suggested by the effective potential, the perturbations modes are concentrated near the center of the trap for $q^{\prime}<q$ where the giant vortex density is very small. For $q^{\prime}>q$ the density $g_{q^{\prime}}$ is concentrated near the wall since the centrifugal term of the perturbation dominates the $q$-vortex influence. Moreover, this figure is in agreement with our assumption of weak off-diagonal terms made above. Indeed, for $\left|q^{\prime}\right|<q$ the interaction term $g\left(q^{\prime}\right) g\left(2 q-q^{\prime}\right)$ is very small since one of the solution is concentrated near $r \sim 0$ while the other one is only present near $r=1$. However, for $q^{\prime}<-q$ the situation cannot be concluded easily since both term is concentrated near $r=1$.

By minimizing the energy $\delta E_{q, q^{\prime}}(\underline{8})$, we can determine whether the functionnal $\delta F_{q^{\prime}}$ (17) is positive definite for all type of perturbations as function of the frequency $\Omega$. The sign of this free energy for the $q^{\prime}$-perturbation changes for:

$$
\Omega_{c}\left(q^{\prime}\right)=\frac{T_{q}+2 U_{q}-\delta E_{q, q^{\prime}}}{q-q^{\prime}}
$$

It tells us that for $q^{\prime}<q$ the $q^{\prime}$ perturbation mode is stable for $\Omega>\Omega_{c}\left(q^{\prime}\right)$ while for $q^{\prime}>q$ it is stable only for $\Omega<\Omega_{c}\left(q^{\prime}\right)$. The change of inequality is due to the change of sign of $q-q^{\prime}$. We define then two critical curves $\Omega_{\text {low }}\left(q^{\prime}\right)=\Omega_{c}\left(q^{\prime}\right)$ and $\Omega_{h i g h}\left(q^{\prime}\right)=\Omega_{c}\left(2 q-q^{\prime}\right)$ calculated both for $q^{\prime}<q$. For a given $\Omega$, if one can find a $q^{\prime}$ such that $\Omega_{\text {low }}\left(q^{\prime}\right)>\Omega$ then the $q$-vortex cannot exist since it decomposes into more complex vortices configuration. On the other hand if one can find a $q^{\prime}$ such that $\Omega_{h i g h}\left(q^{\prime}\right)<\Omega$, the $q$ vortex is also unstable and new vortices enter in the cloud. Thus the $q$-vortex can only be observed if $\operatorname{Max}_{q^{\prime}<q} \Omega_{\text {low }}\left(q^{\prime}\right)<\operatorname{Min}_{q^{\prime}<q} \Omega_{\text {high }}\left(q^{\prime}\right)$ and in the range $\operatorname{Max}_{q^{\prime}<q} \Omega_{\text {low }}\left(q^{\prime}\right)<\Omega<\operatorname{Min}_{q^{\prime}<q} \Omega_{\text {high }}\left(q^{\prime}\right)$ the $q$-vortex is linearly stable. Figures (6) a) and b) show $\Omega_{\text {low }}\left(q^{\prime}\right)$ and 


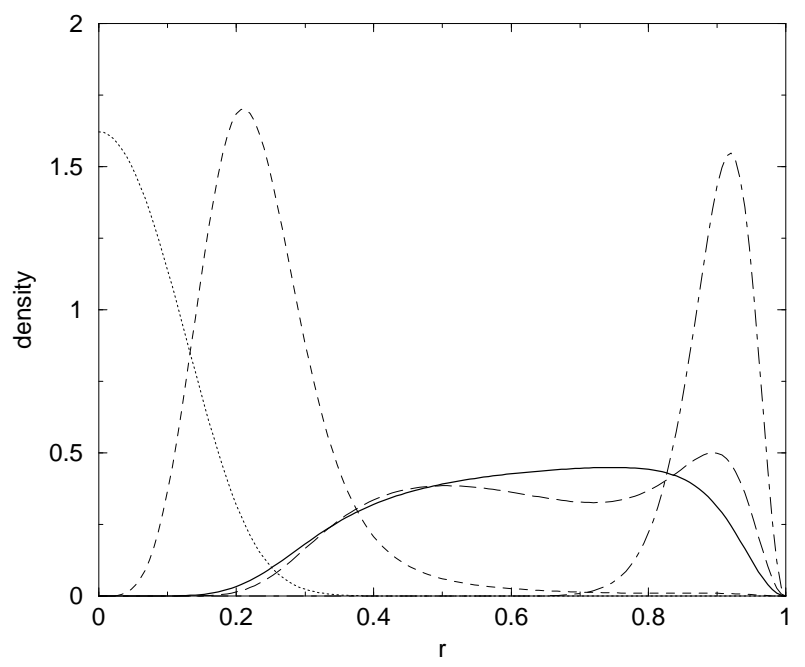

Figure 5: Density profiles of the perturbations modes $g_{q^{\prime}}$ for a $q=4$ giant vortex at $M=300$. Only the profiles for $q^{\prime}=0$ (dotted line), $q^{\prime}=2$ (dashed line), $q^{\prime}=6$ (long dashed) and $q^{\prime}=20$ (dot-dashed) are indicated. The $q=4$ vortex profiles is also shown (solid line). The density for $q^{\prime}=0$ and $q^{\prime}=2$ have been divided by height and two respectively for simplicity.
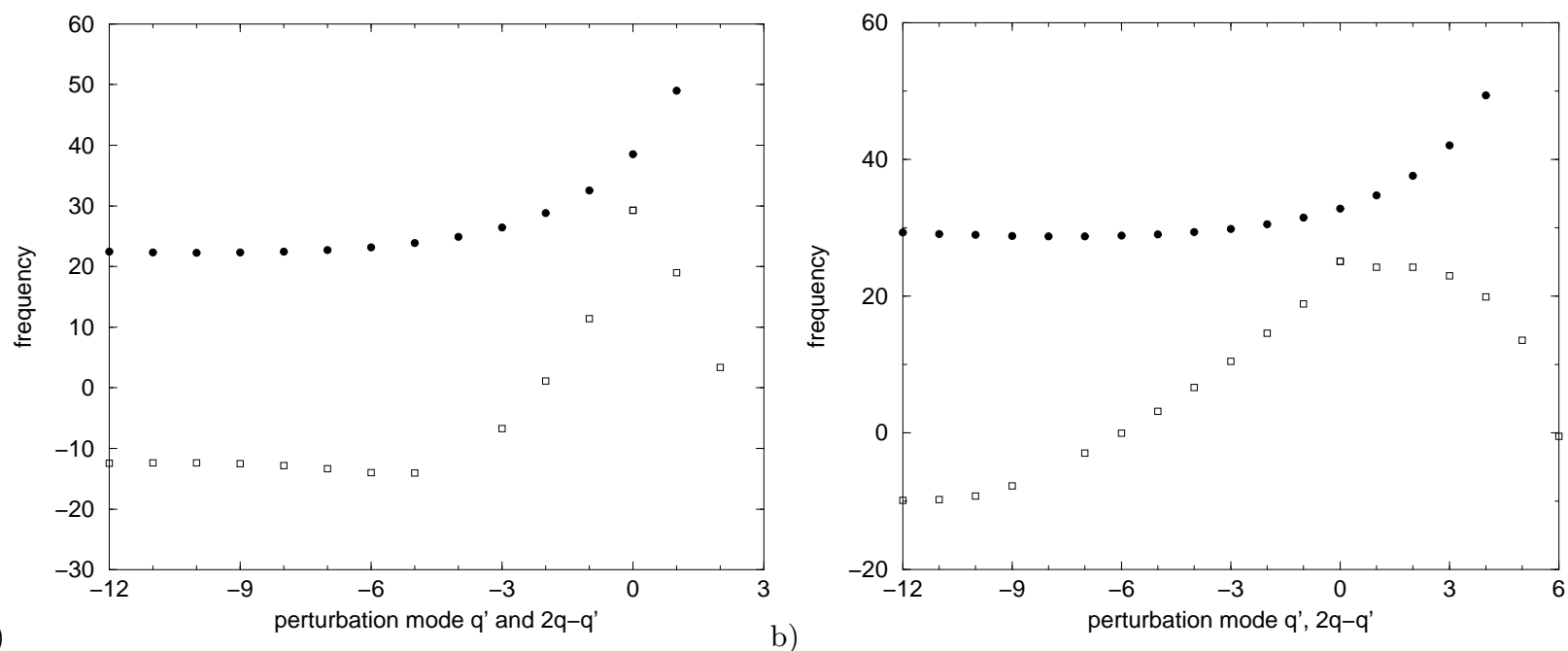

Figure 6: $\Omega_{\text {high }}\left(q^{\prime}\right)$ (black circles) and $\Omega_{\text {low }}$ (squares) as functions of the mode number $q^{\prime}$ for $M=300$, a) $q=4$, and b) $q=8$. It illustrates the two generic situations encountered for the stability of a $q$-giant vortex: a) the 4-giant vortex is never stable; b) the 8-giant vortex is stable for $\Omega$ in the range $[25.09,28.76]$.

$\Omega_{\text {high }}\left(q^{\prime}\right)$ for $M=300, q=4$ and $q=8$ respectively. This figure is found in relatively good agreement with the full numerical simulations of the dynamics shown figure (2). We remark on figure (6) that the 4-vortex cannot be stabilized since the perturbation mode $q^{\prime}=0$ is still unstable when the high vortices perturbation $q^{\prime}=18$ becomes unstable (at $\Omega=22.29$ ) as observed on figure (2). The solution with four single vortices becomes unstable at $\Omega=$ 


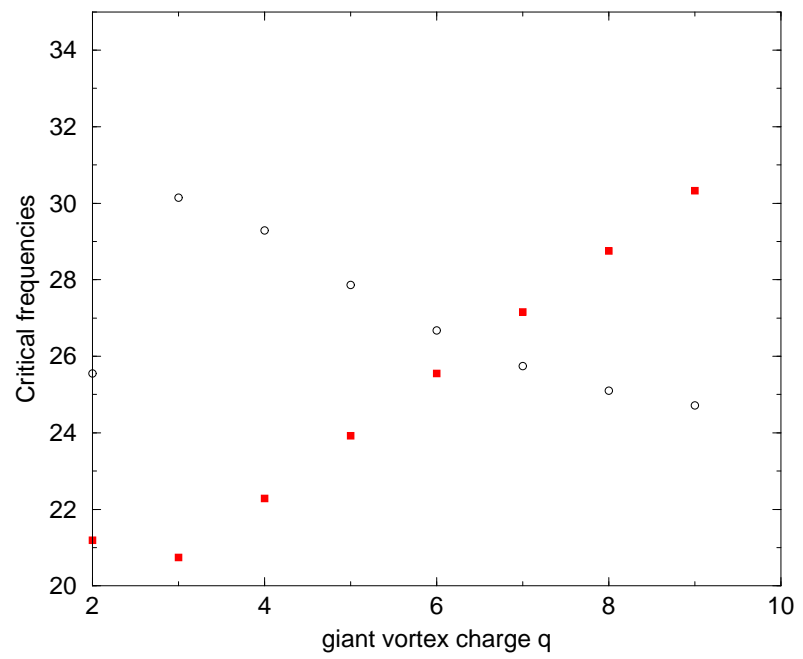

Figure 7: Evolution of $\operatorname{Max}_{q^{\prime}<q} \Omega_{\text {low }}\left(q^{\prime}\right)$ (circles) and $\operatorname{Min}_{q^{\prime}<q} \Omega_{h i g h}\left(q^{\prime}\right)$ (red squares) for the giant vortex charge $q$ between 2 and 15. The two curves cross between $q=6$ and $q=7$, so that only above $\Omega=25.74$ can giant vortices be observed.

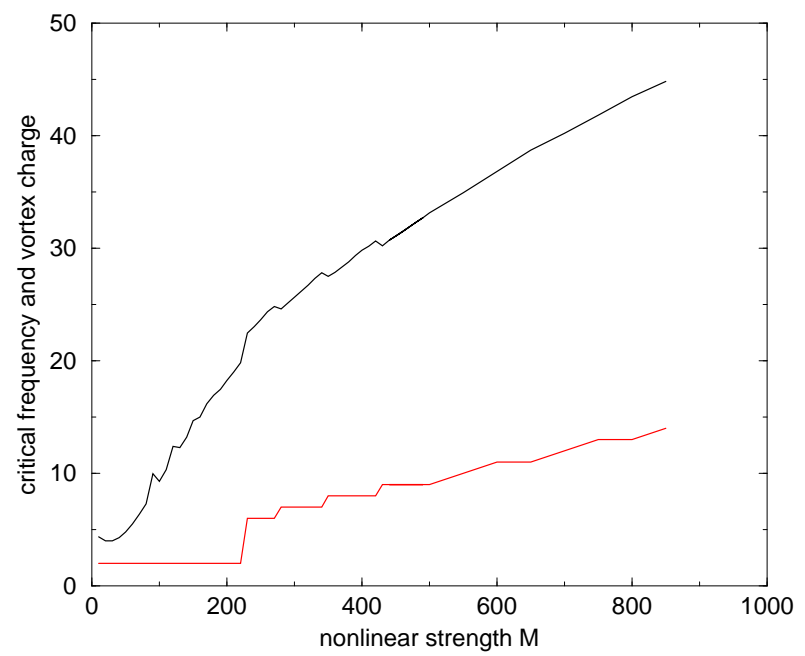

Figure 8: Critical curves $\Omega_{g}(M)$ (black line) and $q_{g}(M)$ (red line). Giant vortices are linearly stable above these curves.

which is close to the value found for the 4 -vortex. For $q=8 \ldots$.

Figure (77) presents the evolution of $\operatorname{Max}_{q^{\prime}<q} \Omega_{l o w}\left(q^{\prime}\right)<\Omega<\operatorname{Min}_{q^{\prime}<q} \Omega_{h i g h}\left(q^{\prime}\right)$ for $q \in[2,15]$. As observed above, it shows that for $q<7$ no giant vortex can be observed while for $\Omega>25.74$ giant vortices of charge $q>6$ can be observed in a given range of $\Omega$.

Defining $\Omega_{g}(M)$ (respectively $\left.q_{g}(M)\right)$ as the lowest frequency (vortex charge) at which a giant vortex can be observed for a strength $M$, we determine in the $\Omega-M$ plane (or $q-M$ plane similarly) the region where the solution consist in stable giant vortices only. The curves $\Omega_{g}(M)$ and $q_{g}(M)$ delimiting these regions are shown on figure (8). 


\section{DISCUSSION}

We have shown numerically that giant vortex solutions can exist in axisymmetrical 2-D BEC model for trap potentials steeper than harmonic. We have investigated in more details the linear stability of these vortices for the cylindrical wall-like potential. The axisymmetry property of the $q$-vortices simplifies the stability procedure into a one dimensional minimization problem. Under a no off-diagonal terms assumption presenting good agreement with the full numerical studies we can determine the regions where the giant vortices are stable. Finally a master curve presenting the frequency and vortex charge above which giant vortices are stable for varying nonlinear strength $M$ is presented. These results have been obtained for the particular case of cylindrical wall potential but can be adapted to smooth BEC potentials. The stability analysis lead to the same minimization problem but the numerical determination of the $q^{\prime}$ modes needs more careful investigations since they are not concentrated on $r \leq 1$ disk.

\section{ACKNOWLEDGEMENTS}

It is my pleasure to thank Vincent Hakim for his encouraging remarks on this work. I also would like thank warmly the editors for organising this special issue. I cannot also finish without express my special thanks to Yves Pomeau who made me discover the magic world of nonlinear dynamics!

[1] C.C. Bradley, C.A. Sackett, and R.G. Hulet, Phys. Rev. Lett. 75, 3969 (1995).

[2] M.H. Anderson, J.R. Ensher, M.R. Matthews, C.E. Wieman, and E.A. Cornell, Science 269, 198 (1995).

[3] K.B. Davis, M.-O. Mewes, M.R. Andrews, N.J. van Druten, D.S. Durfee, D.M. Kurn, and W. Ketterle, Phys. Rev. Lett. 75, 3969 (1995).

[4] R.J. Donnelly, Quantized Vortices in Helium II (Cambridge University Press, Cambridge, England., 1991).

[5] K.W. Madison, F. Chevy, W. Wohlleben, and J. Dalibard, Phys. Rev. Lett. 84, 806 (2000).

[6] C. Raman, M. Khl, R. Onofrio, D. S. Durfee, C. E. Kuklewicz, Z. Hadzibabic and W. Ketterle, Phys. Rev. Lett. 83, 2502 (1999).

[7] R. Onofrio, C. Raman, J. M. Vogels, J. R. Abo-Shaeer,A. P. Chikkatur and W. Ketterle, Phys. Rev. Lett. 85, 2228 (2000).

[8] E.J. Yarmchuk, M.J.V. Gordon, and R.E. Packard, Phys. Rev. Lett. 43, 214 (1979).

[9] T. Frisch, Y. Pomeau and S. Rica, Phys. Rev. Lett. 69, 1644 (1992).

[10] P. Engels, I. Coddington, P.C. Haljan, V. Schweikhard, and E.A. Cornell, cond-mat/0301532 (2003). 
[11] I.S. Aronson and V. Steinberg, Phys. Rev. B 53, 75 (1995).

[12] Y. Castin and R. Dum, Eur. Phys. J. D 7, 399 (1999).

[13] E. Lundh, Phys. Rev. A 65, 043604 (2002).

[14] U.R. Fischer and G. Baym, cond-mat/0111443 (2001).

[15] K. Kasamatsu, M. Tsubota, and M. Ueda, Phys. Rev. A 66, 053606 (2002).

[16] T. Kuga, Y. Torii, N. Shiokawa, T. Hirano, Y. Shimizu, and H. Sasada, Phys. Rev. Lett. 78, 4713 (1997).

[17] E.P. Gross, J. Math. Phys. 4, 195 (1963).

[18] L.P. Pitaevskiŭ, Sov. Phys. JETP 13, 451 (1961).

[19] F. Dalfovo, S. Giorgini, L. Pitaevskiŭ, and S. Stringari, Rev. Mod. Phys. 71, 463 (1999). 